\title{
Kanamycin C
}

National Cancer Institute

\section{Source}

National Cancer Institute. Kanamycin C. NCI Thesaurus. Code C76154.

A minor component of the kanamycin complex, an aminoglycoside antibiotic isolated from Streptomyces kanamyceticus, with antibacterial activity. 